\title{
Heterogeneity at multiple length scales in halide perovskite semiconductors
}

\author{
Elizabeth M. Tennyson, Tiarnan A. S. Doherty and Samuel D. Stranks*
}

Cavendish Laboratory, University of Cambridge, Cambridge, UK.

*e-mail: sds65@cam.ac.uk

\section{TOC blurb}

Despite their excellent macroscopic operational parameters, halide perovskites exhibit heterogeneity in materials properties at all lateral and vertical length scales. In this Review, we discuss the nature of heterogeneity in halide perovskites and assess the impact of these non-uniformities on their optoelectronic properties, and how the heterogeneity may even be beneficial for device properties.

\begin{abstract}
Materials with highly crystalline lattice structures and low defect concentrations have classically been considered essential for high-performance optoelectronic devices. However, the emergence of high-efficiency devices based on halide perovskites is provoking researchers to rethink this traditional picture, as the heterogeneity in several properties within these materials occurs on a series of length scales. Perovskites are typically fabricated crudely through simple processing techniques, which leads to large local fluctuations in defect density, lattice structure, chemistry and bandgap that appear on short length scales $(<100 \mathrm{~nm})$ and across long ranges $(>10 \mu \mathrm{m})$. Despite these variable and complex non-uniformities, perovskites maintain exceptional device efficiencies, and are, as of 2018, the best-performing polycrystalline thin-film solar cell material. In this Review, we highlight the multiple layers of heterogeneity ascertained using high-spatial-resolution methods that provide access to the required length scales. We discuss the impact that the optoelectronic variations have on halide perovskite devices, including the prospect that it is this very disorder that leads to their remarkable power-conversion efficiencies.
\end{abstract}

\section{[H1] Introduction}

Our fundamental semiconductor textbooks dedicate entire chapters to illustrating the detrimental effects of defects in semiconductor devices ${ }^{1-3}$. We are taught and have observed that crystallographic defects such as atoms missing from their nominal lattice positions induce poor electrical conductivity and short charge-carrier lifetimes, and thus that imperfections should be avoided at all costs. However, innovative ways have been found to exploit defects to, for example, shorten carrier lifetimes for fast switching devices or quantum processors $^{4-6}$ and enhance the electrical conductivity in semiconductors through doping ${ }^{4}$. A 
comparatively new class of materials, halide perovskites, have transformed our view of imperfections and impurities, because exceptional optoelectronic performance (the best polycrystalline thin-film perovskite solar cell to date has a power conversion efficiency $(\eta)$ of $24.2 \%)^{7}$ prevails despite large defect concentrations ${ }^{8}$. This apparent paradox for perovskites - that is, exceptionally long carrier lifetimes and diffusion lengths as well as highperformance devices in the presence of many defects ${ }^{9,10}$ - may be related to the fact that many point defects do not lead to deep trap levels ${ }^{11-17}$. However, this paradox may also be a consequence of the hierarchical range of heterogeneity and disorder that pervades throughout these materials. Indeed, the nature of the defects and their presence at multiple length scales are important considerations in explaining the overall material defect tolerance, which is defined here as the ability of a semiconductor to preserve its performance regardless of the defect $_{\text {concentration }}{ }^{17}$. Moreover, perovskites exhibit extraordinary chemical resilience ${ }^{18,19}$ — the high performance of these materials is only slightly perturbed when varying the constituent atoms within the $\mathrm{ABX}_{3}$ lattice structure ${ }^{20,21}$. The primary lattice components in the leading perovskite optoelectronic devices typically comprise a cation, such as methylammonium $\left(\mathrm{MA}^{+}, \mathrm{CH}_{3} \mathrm{NH}_{3}{ }^{+}\right.$), formamidinium $\left(\mathrm{FA}^{+}, \mathrm{CH}\left(\mathrm{NH}_{2}\right)_{2}{ }^{+}\right.$) and/or $\mathrm{Cs}^{+}$; a metal cation, typically $\mathrm{Pb}^{2+}$ and/or $\mathrm{Sn}^{2+}$; and a halide $\left(\mathrm{I}^{-}, \mathrm{Br}^{-}\right.$and/or $\left.\mathrm{Cl}^{-}\right)$. Yet the addition and/or substitution of many other elements, such as $\mathrm{Rb}, \mathrm{K}$ or $\mathrm{Mn}\left(\mathrm{refs}^{22,23}\right)$, typically has no detrimental effect on device operation and can even increase device performance, demonstrating that perovskites are tolerant to a wide range of additives.

These remarkable features of perovskites are further evidenced by comparing the average defect densities and a key figure-of-merit of record perovskite and GaAs laboratory-scale solar cells, as the latter material is a mature light-absorbing semiconductor. For the highly idealized case, GaAs (Fig. 1a), defect concentrations as low as $10^{7} \mathrm{~cm}^{-3}$ can now be achieved $^{24}$. By contrast, for polycrystalline perovskite films, the trap density is $\sim 10^{14}$ 
$10^{16} \mathrm{~cm}^{-3}\left(\mathrm{refs}^{25,26}\right)$, and its single-crystal counterpart maintains defect densities between $10^{9}-$ $10^{11} \mathrm{~cm}^{-3}\left(\right.$ ref. $\left.^{27}\right)$. The impact of non-radiative charge-carrier recombination events on device performance can be assessed through the open-circuit voltage $\left(V_{\mathrm{oc}}\right)^{28}$. For example, the current density-voltage $(J-V)$ characteristics of a high-performance GaAs device ${ }^{29}$ and a solar cell device comprising a passivated $\left(\mathrm{FA}_{0.85} \mathrm{MA}_{0.15}\right) \mathrm{PbI}_{3}$ perovskite ${ }^{30}$ are compared in Fig. 1a. The GaAs device has a $V_{\text {oc }}$ of $1.122 \mathrm{~V}$ with an electronic bandgap of $1.42 \mathrm{eV}$ (ref. ${ }^{31}$ ), which implies that the voltage loss from that given by the Shockley-Queisser limit (that is, the maximum $V_{\text {oc }}$ for a single $\mathrm{p}-\mathrm{n}$ junction semiconductor $)^{32}$ is $\sim 40 \mathrm{mV}$ (ref. $^{28}$ ). The passivated perovskite device has a $V_{\mathrm{oc}}$ of $1.18 \mathrm{~V}$ with a bandgap of $\sim 1.53 \mathrm{eV}$ and therefore exhibits a voltage loss of $\sim 70 \mathrm{mV}\left(\right.$ ref. $\left.^{30}\right)$. However, despite the similar macroscopic $V_{\text {oc }}$ losses, stark differences are typically observed when imaging the local changes in the $V_{\mathrm{oc}}$ of GaAs and perovskite devices at the nanoscale (Fig. 1b,c). GaAs, a material that is epitaxially grown, displays a very uniform local $V_{\mathrm{oc}}\left(\right.$ ref. $\left.^{33}\right)$ (Fig. 1b), which is indicative of excellent defect management. By contrast, $\left(\mathrm{Cs}_{0.05} \mathrm{FA}_{0.8} \mathrm{MA}_{0.15}\right) \mathrm{Pb}\left(\mathrm{I}_{0.83} \mathrm{Br}_{0.17}\right)_{3}$, a perovskite with a device performance comparable to that shown in Fig. 1a, shows voltage variations on lateral distance scales that are equivalent to or larger than the apparent grain size of $\sim 100-200 \mathrm{~nm}$ $\left(\right.$ ref. $\left.^{34}\right)$.

Halide perovskite heterogeneity is relevant to the overall output performance of devices at all length scales and directions (that is, both lateral and vertical; Fig. 2). In this Review, we highlight the nature of heterogeneity that has been observed in halide perovskite thin films and crystals for optoelectronic applications. We discuss recent studies that have revealed the multiple length scales on which property variations are found, including sub-grain $(<100 \mathrm{~nm})$, grain-to-grain $(100 \mathrm{~nm}$ to $10 \mu \mathrm{m})$ and long-range $(>10 \mu \mathrm{m})$ features in both the lateral and vertical directions. Then, we connect the heterogeneity at multiple length scales and propose avenues to exploit it by considering routes towards strain management, interface and/or 
passivation engineering, and large-grain growth mechanisms. We also provide a roadmap for how multi-modal microscopy approaches will provide insight to better understand, control and exploit perovskite heterogeneity. The term 'grain' is defined throughout the Review on the basis of the morphological grain boundary features observed in scanning electron microscopy (SEM) and atomic force microscopy (AFM) images of halide perovskites, giving an 'apparent grain size'. However, we note that diffraction-based measurements are necessary to strictly identify grains and boundaries, as discussed further below.

\section{[H1] Different scales of heterogeneity}

On the individual and sub-grain level $(<100 \mathrm{~nm}$, Fig. 2a), studies suggest that grains contain sub-grain features such as crystallites and twin domains that may be related to local strain fields; these features may alter the diffusion pathways of electrons and ions ${ }^{35-38}$. At longer length scales $(>100 \mathrm{~nm}$ to $<10 \mu \mathrm{m}$, Fig. $2 \mathrm{~b})$, the interfaces between grains and their overall 3D distribution throughout the microstructure of a halide perovskite sample undoubtedly influence non-radiative recombination events, ion migration and the infiltration of species such as oxygen and moisture ${ }^{39-41}$. Some of the encompassing long-range disorder (in the range of tens of micrometres or larger) may stem from these nanoscale and mesoscale heterogeneities; however, undesirable disorder can also arise from unoptimized fabrication practices $^{42}$, sub-optimal interfaces ${ }^{43}$ or poor heat-transfer management that promotes widespread strain or wrinkling ${ }^{44,45}$ (Fig. 2c). These undesirable disorder-related features also manifest themselves in the vertical grain morphology (Fig. 2d) and device operation, resulting in phenomena such as electrical hysteresis ${ }^{46,47}$. In this section, we highlight recent literature that reveals heterogeneity in halide perovskites, beginning down at the sub-grain level and then expanding out across many grains. 


\section{[H2] Sub-grain heterogeneity.}

The structural properties of solution-processed perovskites are determined by the nonuniform distribution of nucleation sites during sample or device fabrication ${ }^{48}$. The quality of the perovskite crystals is dependent on the growth parameters, including the substrate temperature and atmospheric conditions ${ }^{49-52}$; nevertheless, regardless of the synthetic route, halide perovskite grains are, on average, typically smaller than $1 \mu \mathrm{m}$. Within these grains, there is heterogeneity in the electronic, chemical, photophysical and structural properties on a lateral scale of tens of nanometres ${ }^{38,53-59}$. A few recent studies ${ }^{37,38,60}$ suggest that these multiproperty heterogeneities are interrelated and could influence the grain-to-grain and macroscopic performance of perovskite devices, as we discuss below.

To resolve the local electrical response at the nanoscale, conductive atomic force microscopy (c-AFM) has been used to map the $V_{\text {oc }}$, short-circuit current $\left(I_{\mathrm{sc}}\right)$ and the darkdrift current of $\mathrm{MAPbI}_{3-x} \mathrm{Cl}_{x}$ (ref. ${ }^{61}$ ). Substantial inter-grain and intra-grain variations in the $V_{\mathrm{oc}}$ and $I_{\mathrm{sc}}$ are observed and appear to be dependent on the facets of individual grains (Fig. 3a). In regions of certain grains, with particular facets, the combination of both high $V_{\text {oc }}$ and $I_{\mathrm{sc}}$ could contribute to the local photovoltaic performance approaching theoretical limits; however, in many other regions, the performance is substantially lower. Subsequent studies have shown that the heterogeneity in current from c-AFM measurements is also influenced by how effectively the tip extracts charge-carriers; the heterogeneity can be partially mitigated by using an AFM probe that extracts current well ${ }^{62}$. Nevertheless, there is still underlying heterogeneity in $V_{\mathrm{oc}}$ and $I_{\mathrm{sc}}$, and this may be related to specific facet or crystallographic features. Thus, it could in principle be possible to improve the macroscale performance of halide perovskite devices by eliminating local variations in $V_{\mathrm{oc}}$ through engineering materials to grow in preferred orientations or with preferred grain alignments. 
The specific beneficial or detrimental sub-grain orientations remain a captivating open question.

Crystallographic twinning occurs when multiple domains of a crystal are oriented relative to one another according to a symmetry rule known as the twin law ${ }^{63}$. Twinned crystals are typically produced during growth but also occur as a result of a phase transformation or structural deformation. In halide perovskites, the debate in the community around twins has, to date, primarily focused on their potential ferroelectric and/or ferroelastic nature ${ }^{64-66}$, although there has been a recent focus specifically on twin boundaries and the effect that they could have on material properties at the sub-grain level ${ }^{35}$. For example, density functional theory calculations were used to investigate the effect of $\{111\}$ twin boundaries on the electronic properties of two different perovskite systems: pure $\mathrm{FAPbI}_{3}$ and $\mathrm{FA}_{0.875} \mathrm{Cs}_{0.125} \mathrm{~Pb}\left(\mathrm{I}_{0.666} \mathrm{Br}_{0.333}\right)_{3}\left(\right.$ ref. $\left.^{36}\right)$. In pure $\mathrm{FAPbI}_{3}$, the formation energy of the $\{111\}$ twin boundary at zero temperature is very low; therefore, these defects should readily form in real materials at higher temperatures. However, the electronic properties of these twins are relatively benign for single-junction photovoltaic applications, presenting only a small barrier to inter-boundary electron and hole transport. By contrast, in the mixed-cation, mixed-halide perovskite $\mathrm{FA}_{0.875} \mathrm{Cs}_{0.125} \mathrm{~Pb}\left(\mathrm{I}_{0.666} \mathrm{Br}_{0.333}\right)_{3}$, which is suitable for tandem solar cells, twin boundaries induce segregation of $\mathrm{I}^{-}$and $\mathrm{Cs}^{+}$(Fig. 3b, top), leading to a local valence band maximum that is $0.2 \mathrm{eV}$ higher in energy that that of the bulk (Fig. 3b, bottom). Such heterogeneous valence band energies could lead to holes preferentially collecting near the twin boundaries, increasing the number of electron-hole recombination events in these regions and ultimately influencing device performance. We note that twins often form in a material in response to a local strain. In light of recent reports that have revealed the impact of strain on the photophysical properties of perovskites ${ }^{38,60}$, the presence of twins may be associated with a strained defective region, even if merely as a benign indicator. 
The theoretical prediction of twin boundaries and their impact on optoelectronic properties in mixed cation and halide perovskites motivates the need for a more thorough experimental understanding of structural sub-grain heterogeneity through crystallographic diffractionbased approaches. This knowledge will be essential to optimize materials synthesis, such as the development of deposition methods that mitigate or modulate the formation of undesirable sub-grain crystallographic boundaries. However, there have been only a limited number of sub-grain crystallographic studies to date, highlighting the difficulty in acquiring high-quality information on the nanoscale structural heterogeneity in halide perovskites, which are much more beam-sensitive than comparable inorganic semiconductors ${ }^{67}$. For example, in electron microscopy, the structure of $\mathrm{MAPbI}_{3}$ can be substantially altered even under low electron doses. These structural changes result in twin domains vanishing ${ }^{35}$, the homogenization of image contrast and the degradation of the tetragonal phase into first a $\mathrm{MAPbI}_{2.5}$ supercell and then $\mathrm{PbI}_{2}\left(\right.$ refs $\left.^{53,68}\right)$. In electron diffraction studies, this degradation manifests as the disappearance of expected diffraction spots, or reflections, and the emergence of additional forbidden reflections. These observations signify that the intrinsic nanoscale heterogeneity of pristine halide perovskites can be masked with improper beam conditions. When performing electron microscopy experiments, the total accumulated electron dose is the important factor to consider, and cumulative doses below the damage threshold of $\sim 100 \mathrm{e}^{-} \AA^{-2}$ are essential to successfully mitigate beam damage in $\mathrm{MAPbI}_{3}$ $\left(\operatorname{refs}^{53,68}\right)$. These dosage guidelines have been established specifically for transmission electron microscopy performed at moderately high accelerating voltages $(\sim 80-200 \mathrm{keV})$. Although beam damage mechanisms vary with the energy of the incident electrons ${ }^{69}$, a study on beam damage at lower accelerating voltages $(\sim 4.5-60 \mathrm{eV})$ indicates that the damage is still induced at comparable accumulated electron $\operatorname{doses}^{70}$. To add to the complexity, systems that incorporate $\mathrm{Cs}^{+}$as the cation have been found to be more stable under an electron beam than 
variants containing $\mathrm{FA}^{+}$or $\mathrm{MA}^{+}$only ${ }^{71}$. It is likely that each new composition will have different tolerable dose limits, and thus as the composition and structure of state-of-the-art perovskite materials continue to evolve, we must be rigorous in the evaluation of how our experiments modify materials and establish sensible frameworks for characterization.

New approaches to locate crystal zone axes, image alignment and the accurate determination of the defocus value in a transmission electron microscopy map have recently enabled atomic spatial resolution of $\mathrm{MAPbI}_{3}$ single crystals with a total accumulated electron dose of $\sim 11 \mathrm{e}^{-} \AA^{-2}$ (ref. $^{67}$ ). Different orientations of the $\mathrm{MA}^{+}$cations are observed across a $12 \mathrm{~nm}$ region (Fig. 3c). Relative to the projection direction, the organic cations adopt normal and parallel orientations, which induces both in-plane and out-of-plane electric dipoles, respectively, and suggests that $\mathrm{MAPbI}_{3}$ exhibits ferroelectric order ${ }^{72}$. Ferroelectricity in metal halide perovskites has been proposed as a mechanism for hysteresis as well as increased performance through enhanced charge-carrier separation and transport ${ }^{68,72-74}$. However, the presence of ferroelectricity in halide perovskites remains a topic of ongoing debate and the answer may depend on several factors, including composition and local structural properties $^{65,66,75}$.

There have also been reports of ferroelasticity in halide perovskites ${ }^{64}$. Ferroelasticity is a well-reported phenomenon in oxide perovskites and, similar to ferroelectricity, can be related to the nanoscale structural and compositional heterogeneity. This phenomenon is characterized by an elastic hysteresis that results from the mechanical switching between two crystallographic orientations owing to the application of an external stress ${ }^{76}$. Inherent coupling between ferroelasticity and local chemical segregation within $\mathrm{MAPbI}_{3}$ grains (Fig. 3d) has been revealed using several force microscopy and compositional mapping techniques ${ }^{37}$. Piezoresponse force microscopy measurements show striped domains that are assigned as ferroelastic twin regions (Fig. 3d, top), which exhibit local ion segregation (Fig. 
$3 \mathrm{~d}$, bottom). It is proposed that this chemical segregation is induced by the release of inherent strain energy from the sample. Furthermore, these periodically ordered, chemically segregated domains may also possess different crystallographic orientations, and these combined properties are a signature of ferroelastic behaviour. This chemical heterogeneity on the sub-grain scale could have notable positive or negative effects on the electronic properties of halide perovskites. For example, the heterogeneity could either enhance charge-carrier separation near the domain walls owing to ion segregation and thus improve photovoltaic properties or limit device performance owing to the presence of low-conductivity domains ${ }^{37}$.

\section{[H2] Grain-to-grain heterogeneity.}

The variations in the properties of halide perovskites expand beyond the sub-grain length scale to their encompassing grains. The most commonly reported grain sizes in highperformance halide perovskite devices are between $100 \mathrm{~nm}$ and $1 \mu \mathrm{m}$; therefore, grain-tograin heterogeneity can be readily visualized using advanced metrology techniques. Interestingly, pure morphological microscopy studies, such as those using scanning electron microscopy (SEM) imaging and AFM topography mapping, of the constituent halide perovskite grains, suggest that the physical properties of the grains are isotropic. However, upon implementing functional imaging or diffraction-based approaches, the grain-to-grain variation is found to be non-uniform, unpredictable and enlightening (for example, Fig. 1c), as demonstrated in this section.

Confocal photoluminescence (PL) microscopy is a powerful technique to observe local heterogeneity in charge-carrier recombination ${ }^{77}$. However, the local nature of both excitation and emission collection means that diffusion of charge carriers out of the probed region can complicate the interpretation of results. To decouple the influence of trapping and diffusion, a comparison of the confocal $\mathrm{PL}$ response of a $\mathrm{MAPbI}_{3}$ perovskite thin film subjected to 
various incident light intensities was performed ${ }^{78}$. On comparing the PL maps at low ( 3 suns; Fig. 4a, left) and high (1,500 suns; Fig. 4a, right) excitation intensities, two immediate qualitative differences are evident: first, the PL heterogeneity is reduced upon exposure to the higher photon concentration, and second, some perovskite grains only emit efficiently under high-fluence conditions. It is concluded that trap-mediated pathways become saturated at higher excitation densities and thus the PL response is dominated by charge-carrier diffusion. Therefore, at sufficiently low excitation densities, such as solar-relevant photon fluxes, the PL distribution is typically dominated by trap-assisted recombination, and it is possible to ascertain trap-related heterogeneity from such measurements. These results highlight the complicated interplay between charge-carrier diffusion and trapping. The measurements also imply that there remains substantial non-radiative heterogeneity in halide perovskite films that needs to be further eliminated through improved processing or surface passivation approaches.

The observation of charge-carrier recombination heterogeneity (Fig. 4a) provides strong evidence that the halide perovskite microstructure, composed of grains and grain boundaries, affects the overall optoelectronic properties and device performance. It is therefore understandable that perovskites with larger grain sizes are being targeted as a route to mitigate non-radiative recombination losses ${ }^{39,77,79-81} . \mathrm{MAPbBr}_{3}$ perovskite thin films with grain sizes in the range $1-60 \mu \mathrm{m}$ were fabricated ${ }^{82,83}$ and characterized using SEM (Fig. $4 \mathrm{~b}$, top) and electron back-scattering diffraction (EBSD) (Fig. 4b, bottom). EBSD is a powerful technique that provides crystallographic information for each constituent grain within a sample. This characterization method has previously been inaccessible for halide perovskite materials owing to the high electron doses required. However, by using a highly sensitive solid-state detector, EBSD maps could be acquired with electron doses that were 6,000 times lower than those required in traditional EBSD approaches, albeit still above the nominal 
damage thresholds for $\mathrm{MAPbI}_{3}\left(\right.$ ref. $^{82}$ ). The EBSD measurements revealed that the sample with an average grain size of $32 \mu \mathrm{m}$ exhibits a disordered region at each of its grain boundaries (indicated by the white regions in the bottom middle part of Fig. 4b). It is proposed that these unidentifiable areas are amorphous and, despite the lack of structural information, were found to be are highly emissive ${ }^{84}$. On the basis of the distribution of grain orientations for each perovskite sample, the majority of the crystals are oriented along the [001] direction, but the heterogeneity of the crystallographic orientation increases with larger grain sizes. The recombination velocities are mostly higher at grain boundaries than those within grains, which in turn negatively affects the macroscopic device performance. Overall, it is concluded that increasing the grain size to the millimetre scale while simultaneously reducing the recombination velocity will push halide perovskites towards their theoretical optoelectronic performance limits ${ }^{85}$.

Time-resolved microwave conductivity (TRMC) measurements, which monitor the alternating current charge mobility as a function of $\mathrm{MAPbI}_{3}$ grain size $^{86}$ (Fig. 4c), also revealed that larger grains would enhance perovskite device efficiency. Samples with a larger density of grain boundaries (that is, a small grain size) exhibited more limited carrier transport, and only when the grain size exceeds $1 \mu \mathrm{m}$ does the mobility approach the intrinsic mobility value of $29 \mathrm{~cm}^{2} \mathrm{~V}^{-1} \mathrm{~s}^{-1}$ at the probe frequency of $8.9 \mathrm{GHz}\left(\right.$ ref. ${ }^{86}$ ). These results will need to be reconciled with recent device studies that show that the solar cells with the highest performance to date exploit mixed cation systems, which have relatively small grain sizes; it is likely that the precise nature of the grain boundaries is more crucial than the actual grain size.

Luminescence microscopy approaches to study local heterogeneities are typically only capable of probing the surface or first few nanometres in the depth of a sample. One option to examine the interior of a halide perovskite layer is to perform cross-sectional 
measurements ${ }^{46,87}$; however, these approaches are often destructive. With two-photon PL microscopy ${ }^{88}$, it is possible to probe deeper into a semiconductor material without damaging the sample by using two low-energy photons (each with an energy lower than the bandgap). These low-energy photons impinge deeper into the sample than the high-energy photons and only photoexcite the material in a small focused volume. Depth-dependent studies are of wide interest to the halide perovskite device community owing to the multiple heterojunctions formed at the interfaces with the electron-transport and hole-transport layers ${ }^{43,89}$, variations in recombination as well as charge and defect transport with depth ${ }^{90}$, and ion migration ${ }^{91}$. Recently, two-photon PL and single-photon (conventional) PL microscopy were performed on the same scan region of a $\mathrm{MAPbI}_{3}$ sample $^{92}$ (Fig. 4d). The one-photon excitation PL map (Fig. 4d, left) was acquired with a 510-nm pulsed laser, which was used to selectively generate photoexcitations near the surface of the film, whereas the two-photon PL map (Fig. $4 \mathrm{~d}$, right), was obtained using a 1,100-nm pulsed laser, which generates photoexcitations further into the bulk. Comparing the two maps, some grains appear brighter at the surface than in the bulk (indicated by the red circles in Fig. 4d), while other grain clusters demonstrate the opposite phenomenon (indicated by the green circles in Fig. 4d). Such depthdependent PL results reveal buried recombination processes, which suggest that there is both lateral and vertical heterogeneity in the radiative recombination. Thus, the depth-dependent charge-carrier recombination may lead to undesirable performance deficiencies such as unbalanced electronic transport rates $^{93}$ and charge-collection and/or trapping issues in devices.

Imaging within perovskites is also crucial for understanding one of the biggest problems associated with mixed-halide compositions: local phase segregation ${ }^{94}$. Varying the halide composition, usually by mixing $\mathrm{Br}^{-}$and $\mathrm{I}^{-}$, is a common approach to tune the bandgap of perovskites ${ }^{95-97}$. When an external stimulus (typically, incident light) is applied to a mixed- 
halide perovskite, $\mathrm{Br}^{-}$and $\mathrm{I}^{-}$ions segregate into local $\mathrm{Br}^{-}$-rich or $\mathrm{I}^{-}$-rich clusters ${ }^{94}$. This chemical segregation results in variations in the electronic bandgap ${ }^{98-102}$. The lateral distribution of the bandgap in a $\mathrm{CsPbIBr}_{2}$ film has been visualized using spectrally and spatially resolved cathodoluminescence (CL) imaging ${ }^{103}$. The all-inorganic $\mathrm{CsPbIBr}_{2}$ perovskite was studied owing to its higher thermal stability and greater resistance to electron beam damage than its organic analogues ${ }^{71,104}$. Grains and grain boundaries are clearly identifiable in the spectrally integrated CL maps of emission from $530-590 \mathrm{~nm}$ (corresponding to the $\mathrm{Br}^{-}$-rich phase; Fig. 4e, top) and from 590-640 nm (corresponding to the $\mathrm{I}^{-}$-rich phase; Fig. 4e, middle). From the superposition of the two CL maps (Fig. 4e, bottom), it is apparent that $\mathrm{I}^{-}$-rich regions are preferentially distributed at the grain boundaries; absolute CL maps also demonstrate that these regions are more emissive than the grain interior. The preferential distribution may indicate that the $\mathrm{I}^{-}$-rich domains are locally confined, which leads to higher charge-carrier densities and subsequently enhanced local radiative recombination. The brighter emission at the grain boundaries may also be due to the transfer (funnelling) of charge carriers to a phase with a lower bandgap ${ }^{105}$.

The CL maps (Fig. 4e) enable comparison of the material properties of the grain interior to those at the grain boundaries. Although there is general consensus that low concentrations of grain boundaries are conducive to better device performance, there is also evidence that not all grain boundaries are detrimental to photovoltaic device performance ${ }^{79,106}$. Furthermore, it is likely that not all grain boundaries influence performance in the same way, as evidenced in confocal fluorescence-lifetime imaging microscopy (FLIM) maps of a $\mathrm{MAPbI}_{3}$ perovskite thin film ${ }^{79}$ (Fig. 4f). Scans of FLIM intensity and lifetime across three distinct grains (of similar diameters) show that there is not a clear correlation between the PL emission intensity and charge-carrier lifetime. In this case, the PL intensities of the selected grains are lower than those of the neighbouring grains (Fig. 4f, right), yet the charge-carrier lifetimes are 
either locally maintained, extended or reduced. It is also clear that there is a wide grain-tograin distribution of both the PL intensity and lifetime. Note that the carrier lifetime and resulting PL intensity will depend strongly on the local charge-carrier and trap density, which ultimately dictate the dominant recombination regime (trap limited or radiative bimolecular $)^{28,107,108}$. Therefore, the grain boundaries in this study might exhibit similar behaviour but differ in their local absolute trap density. It is also possible that grain boundaries are benign in terms of promoting recombination but prevent charges from diffusing between grains; thus, the carriers are confined to a specific grain, resulting in each grain having specific electrical and/or optical properties. This confinement may depend on the composition of the grain boundary; for example, an excess of a material with a large bandgap, such as $\mathrm{PbI}_{2}$, at the grain boundaries may retain charges within a halide perovskite grain, but other defects in this region, such as ionic vacancies or interstitials, might promote non-radiative recombination. In general, it is likely that the properties of grain boundaries are very specific to the composition and even fabrication method ${ }^{77}$; thus, grain boundaries cannot be universally defined as being detrimental or benign.

\section{[H2] Long-range heterogeneity.}

Long-range variations in the properties of semiconductor materials are frequently observed using optical microscopy (reflection or transmission) ${ }^{40,75,109}$, wide-field PL and electroluminescence $(E L)^{44,110}$ or low-magnification SEM imaging modes ${ }^{111}$. To alleviate long-range non-uniformities in material properties, there have been notable advances in device fabrication and synthesis techniques to increase the connectivity of the perovskite grains for improved film quality ${ }^{83,112-119}$. Eliminating the long-range heterogeneity will likely have a substantial impact on device performance, such as pushing the $V_{\text {oc }}$ towards the radiative limit ${ }^{32}$ and overcoming problems associated with series and shunt resistance. 
However, the eradication of long-range heterogeneity may also remove the disorder that possibly endows halide perovskites with some of their unique properties.

The $V_{\mathrm{oc}}$ of a solar cell can be quantified by measuring the external quantum efficiency for EL (which is directly related to the photovoltage) and the EL spectrum ${ }^{120,121}$. However, typical polycrystalline perovskite films show heterogeneous charge-carrier radiative recombination and therefore exhibit non-uniform photovoltage distributions (Fig. 1c). A perovskite solar cell was characterized using wide-field hyperspectral imaging, which enabled absolute EL and PL maps to be generated through the generalized Planck's law ${ }^{122}$ and the local quasi-Fermi level splitting ( $\Delta \mu$, which is proportional to the $V_{o c}$; Fig. 5a) to be estimated $^{110}$. In the $\Delta \mu$ map, there are dark spots that are tens of micrometres in size. These spots are most likely a consequence of imperfect electrical connections between the electroncollecting layer $\left([6,6]\right.$-phenyl $\mathrm{C}_{61}$-butyric acid methyl ester) and the perovskite $\left(\mathrm{MAPbI}_{3}\right)$ layer and demonstrate that there are both local and long-range charge-carrier collection issues. The larger variations in $\Delta \mu$ may be due to lateral charge-carrier transport losses and local fluctuations of the interfacial series resistance. It is also possible that the film is nonuniform (for example, that there are variations in thickness and/or coverage), which could lead to the formation of pinholes and other imperfections in the film that reduce the device performance. Such heterogeneity in quasi-Fermi level splitting can be minimized through judicious use of thin interlayers between the perovskite and transport layers ${ }^{123}$.

Structural non-uniformities, such as preferred crystallographic orientations and long-range strain fields, may also contribute to variations in optoelectronic properties. These structural non-uniformities greatly affect the overall performance of mature semiconductors materials, but, to date, have been under-investigated in halide perovskites ${ }^{45,124,125}$. Recently, micro Xray diffraction $(\mu \mathrm{XRD})$ and time-resolved confocal PL imaging were performed on the same scan regions of halide perovskite thin films to visualize how strain varies on the microscale 
and affects the radiative recombination of carriers ${ }^{38}$. By tracking local fluctuations in either the position or broadness of diffraction peaks in $\mu \mathrm{XRD}$, strain-induced variations in the interatomic spacing of a material can be observed. In a $\mathrm{MAPbI}_{3}$ film, the PL lifetime traces from poorly and brightly emissive regions (Fig. 5b, left) correspond to regions of higher and lower compressive strain, respectively, in the compressive strain map extracted from $\mu \mathrm{XRD}$ measurements (Fig. 5b, right; the red and blue circles indicate the regions from which the local PL decays were extracted). As the less strained (brightly emissive) regions have significantly longer lifetimes than the highly strained areas, it indicates that the local strain is associated with trap-related non-radiative recombination events. Further nano-XRD experiments on these $\mathrm{MAPbI}_{3}$ samples revealed 'super-grain' structures (defined here as regions with common azimuthal scattering angles) as large as $25 \mu \mathrm{m}^{2}$ in lateral area ${ }^{38,126}$, signifying long-range structurally uniform regions at least an order of magnitude larger than the average grain sizes identified by SEM. Surprisingly, a film of an alloyed cation perovskite, which on average has better optoelectronic performance (for example, solar cell $\eta$ ) than $\mathrm{MAPbI}_{3}\left(\right.$ ref. $^{127}$ ), contains super-grains that are up to $\sim 250 \mu \mathrm{m}^{2}$. Despite the inherently smaller grain size within the alloyed cation sample, determined by SEM micrographs, it is possible that the long-range structural super-grains contribute to the improved performance in the alloyed cation devices. This finding also raises the question of which structural grain size 'unit' is most important for device performance.

To control and tune the grain size, wrinkling and other structural features of halide perovskite films, it is important to have complete understanding of the crystal nucleation mechanisms and growth parameters ${ }^{128-130}$. In this regard, embedding perovskite (in this case $\mathrm{Cs}_{0.10} \mathrm{FA}_{0.78} \mathrm{MA}_{0.12} \mathrm{PbI}_{2.55} \mathrm{Br}_{0.45}$ ) seed crystals into a $\mathrm{PbI}_{2}$ matrix during a two-step sequential deposition was shown to enable a high level of control over perovskite crystallization ${ }^{131}$. The seeds facilitate the reaction of organic ammonium halides with $\mathrm{PbI}_{2}$ and thereby act as nuclei 
for the subsequent growth of the perovskite film. In situ PL imaging was used to monitor perovskite crystallization during the growth process (Fig. 5c). Five seconds after the deposition of the organic ammonium salts onto the seed- $-\mathrm{PbI}_{2}$ film (Fig. 5c, middle), two distinct types of growth kinetics emerge: first, perovskite-seed-assisted growth (indicated by the white circles on the map), and, second, random nucleation without seeds (indicated by the red dashed circle). The perovskite-seed-assisted growth kinetics appears faster than that of random nucleation without seeds, and a later snapshot (Fig. 5c, right) demonstrates how the perovskite-seed-assisted growth dominates crystallization. The results highlighted here suggest that this deposition technique could be further optimized and extended to other substrate and transport layer combinations for improved growth control of perovskite films.

Almost independent of the deposition technique, halide perovskites still exhibit surprisingly long diffusion lengths ${ }^{10}$ that extend well beyond individual perovskite grains. For example, long diffusion lengths were recently observed in $\mathrm{MAPbBr}_{3}$ conjoined microcrystals in which grains were fused, enabling effective transport of charge carriers between grains ${ }^{132}$. These fused grains exhibited synced intermittent fluorescence blinking, which can be observed in the PL map and integrated PL time traces (Fig. 5d). The synchronous communication between photogenerated charge carriers extends as far as $\sim 10 \mu \mathrm{m}^{2}$ laterally. It is proposed that the prominent fluctuations in PL intensity are at least partly due to the creation and suppression of defects, which temporally quench emission near these regions. Similar connectivity of optoelectronic properties between grains has also been reported for $\mathrm{MAPbBr}_{3}$ thin films ${ }^{133}$. These studies suggest that the relationship between defects and charge energy-transfer are complex ${ }^{62,110,134-138}$. Although these specific PL responses related to grain connectivity are primarily relevant when the perovskite film displays discontinuities and voids, these responses demonstrate that charge carriers will experience various grain 'environments' during their lifetime. Owing to the observed large lateral carrier and/or defect 
transport distances, a range of transport and annihilation mechanisms, such as photon recycling, reabsorption, waveguiding, carrier diffusion, defect passivation and drift, must be considered to understand the PL response. For example, it is suggested that blinking may not be related to charge transport but could instead be a consequence of surface modification or passivation of the halide perovskite owing to fluctuations of the ambient environment (for example, moisture, oxygen content or temperature). Photon recycling events can also lead to rapid apparent transport of carriers over long distances through reabsorption and re-emission of photons at a distance of several micrometres away from the original excitation ${ }^{139,140}$.

\section{[H1] Leveraging disorder}

\section{[H2] Connecting the length scales.}

Upon stepping back and assessing the observations reviewed herein, we are left with one central question: how do the heterogeneities at multiple length scales interrelate? To address this question, we start by considering halide perovskites in terms of their material properties. At the atomic scale and nanoscale (that is, the sub-grain level), strain is induced by changes or distortions in the lattice structure, primarily due to point, line, planar and interfacial defects. In each case, the strain and structural properties are related to the atomic (elemental) distribution, resulting in an intricate relationship between the local chemical and structural heterogeneity, as shown, for example, in Fig. 3d. The properties of halide perovskites are particularly sensitive to this nanoscale chemical distribution, which dictates the local bandgap, connecting the optoelectronic behaviour and nanoscale structural and chemical properties. Owing to the long-range electronic transport of the charge carriers (governed by the regional structural and chemical nature of the material), the electrons and holes will diffuse, dispersing throughout the heterogeneous grain-to-grain landscape. The direction and motion of the charge carriers is dependent on several factors, including bandgap gradients 
and diffusion pathways with low energy barriers. As perovskite grain boundaries can have different electronic properties, there can be preferential transport pathways for charge carriers moving between grains that may also depend on the grain connectivity ${ }^{78}$ (Fig. 4).

In the halide perovskite community, grain boundaries are interpreted as the interfaces between discrete grains identified with electron or optical microscopy. The assumption is that the material within these grains is homogeneous and single-crystalline. However, this interpretation is often too general as it overlooks the manifestation of sub-grain crystallographic misorientations and variations, which require various diffraction-based techniques to fully understand. As an example, (sub-grain) twin boundaries have been proposed to have a notable impact on the electronic, compositional and mechanical properties of semiconducting materials such as GaAs, $\mathrm{Si}$ and even perovskites ${ }^{37,61,141,142}$. To fully understand the operation of polycrystalline perovskite materials at the nanoscale, highresolution diffraction-based approaches are required to reveal whether there are crystallographic heterogeneities within entities that have been assigned as homogenous grains through morphological measurements ${ }^{35,38,82,143}$.

As discussed above, grains sometimes act as isolated systems, or islands, where the termination of common optoelectronic behaviour (for example, PL response) is marked by the presence of a grain boundary. Determining which type(s) of grain boundary causes this abrupt change in optoelectronic response is an active area of research. Indeed, the nature of these boundaries may affect the optoelectronic response more than grain size. For example, a system with reasonably small grain sizes but optimized boundaries could still show excellent optoelectronic properties. This is likely the case in mixed cation systems, which have thus far exhibited the highest performance in solar cells despite the relatively small grain sizes. There is also evidence that these localized property 'transitions' at boundaries may be linked to the underlying super-grain distribution or other long-range strain fields ${ }^{38}$. Therefore, the variation 
in grain-to-grain optoelectronic, chemical and structural properties necessarily influences beyond the individual length scale, manifesting into grain clusters ${ }^{34}$ and long-range nonuniformities, as highlighted in Fig. 5. Once the origin of a specific long-range heterogeneity feature is understood, it will be possible to mitigate or exploit the effect for improved optoelectronic devices.

It is likely that small nanoscale domains in the range of tens of nanometres are building blocks that dictate the longer range heterogeneity (on the order of hundreds of nanometres to tens of micrometres). Ultimately, the nanoscale domains self-assemble from small seeds, and the subsequent grain growth will be influenced by the local growth environment; as grains begin to meld into one another, strain at the grain interfaces influences the quality of the resultant film (analogous to strain-related interactions at the boundaries of two tectonic plates, influencing geologic activity at that interface ${ }^{144}$ ). Elucidation of the interplay between growth mechanism and strain necessitates the use of advanced characterization techniques (for example, morphology, chemical, diffraction and optoelectronic) to monitor properties in situ during growth.

\section{[H2] Levers for understanding heterogeneity.}

Many forms of excitation and detection have been implemented to visualize the properties of halide perovskites at different length scales. External stimuli (stressors) that are commonly used to activate specific perovskite material behaviours and responses include photons $^{34,40,91,105,142,145-159}$, electrons ${ }^{22,87,101,160-162}, \quad$ temperature ${ }^{163-166}$ and $\operatorname{bias}^{46,167,168}$. However, in a typical experiment, only one such stressor is used, which generally limits the investigation to the study a single material property. Moreover, the majority of techniques currently used to probe microscale properties of halide perovskites are based on luminescence; however, a holistic understanding of these materials will require the use of 
complementary techniques beyond luminescence. Therefore, to connect the different length scales of heterogeneity, the perovskite community must also connect a range of characterization methods and external stimuli to probe and correlate distinct material responses. Future studies call for intensive investigations that closely relate the entire narrative of a sub-grain feature to its long-range heterogeneity and performance in operating solar cells (Fig. 6).

Currently, most fundamental studies on halide perovskites are performed on thin-film samples, and there are questions regarding the relevance of these reports to full devices ${ }^{169}$. For example, the optoelectronic properties of perovskites change substantially when a thin film interacts with a device contact ${ }^{28}$, and it is likely that aspects such as morphology, strain and ion distribution will also be influenced by these interfaces. Strain and non-radiative losses will also be altered in a full device configuration, and may even dominate the performance output and operational longevity, albeit, operating times of $>1,000$ hours are currently possible in devices with extensive heterogeneity ${ }^{170}$. Performing the studies reviewed above on half- or full-device structures would forge connections between loss and performance and would allow for a greater diversity of in situ and in operando characterization techniques, such as light-beam and/or electron-beam induced current ${ }^{87,170}$. Another key aspect that has not been sufficiently probed in perovskites, especially in operating devices, is the local composition ${ }^{19}$. From the limited works available, researchers have thus far discovered important fundamental perovskite behaviours, such as the link between processing conditions and the depth distribution of the organic cation ${ }^{171}$, the need to uniformly mix halides for optimal performance ${ }^{60}$, that light induces halide segregation in mixed-halide perovskite compositions ${ }^{105}$, and the necessary solvent and cation precursor stoichiometry to synthesize highly crystalline perovskites of reduced dimensionality ${ }^{172}$. 
Incident light ${ }^{173}$ and oxygen exposure ${ }^{174,175}$ can be applied to mitigate non-radiative decay by passivating defects. Therefore, by using multimodal microscopy techniques to characterize a sample before and after the application of these external stressors, it may be possible to ascertain how non-radiative recombination losses are distributed at different length scales. Light and temperature can, in principle, be used to control ion motion and the degradation of halide perovskitess ${ }^{34,142,166}$; thus, these stimuli enable many local physical properties to be monitored as either a function of ionic distribution or temporal performance variation. We anticipate that multimodal microscopy approaches could be used in conjunction with controlled activation sources to visualize the resultant changes in properties across many length scales.

Such multimodal studies to correlate optoelectronic and/or device properties with the chemical, structural and morphological properties are not trivial and are associated with their own unique set of challenges. These approaches typically require synchrotron-based experiments, such as X-ray nanoprobe or specialized electron microscopy facilities (for example, advanced scanning electron diffraction techniques ${ }^{176}$ combined with direct electron detectors), to achieve the required spatial resolution for probing the desired properties. These facilities are not easily accessible for routine or screening measurements. Furthermore, beam damage under intense electron or X-ray irradiation must be carefully monitored by performing appropriate checks before, during and after an experiment to assess the impact of the measurement on the sample; this is true even if the electron dose is well below the reported damage thresholds, as operating devices may be more susceptible to damage than thin films. Fiducial markers are crucial to ensure sharp image registration between two experiments and to enable precise analysis of the same scan area. These measurements typically generate large data sets (for example, many terabytes of data are generated in a typical nanoprobe map measurement), and thus efficient algorithms and software are required 
to process the large hyperspectral data sets. HyperSpy ${ }^{177}$ and pyXem ${ }^{178}$ are two promising examples of algorithms that are currently being used at some synchrotron and electron microscopy facilities as the demand for multimodal correlations increases. Machine learning is the clear next step to expedite data analysis in these multimodal studies and thus increase the throughput of analysis and characterization ${ }^{179-182}$. Advanced algorithms will substantially improve signal recovery from noisy data (which is particularly pertinent in perovskites as they require low X-ray and electron doses) and enable precise correlations between parameters, which will be the key to discovering new relationships between properties. We believe that a multimodal approach, despite its challenges, will be a vehicle for capitalizing on the heterogeneity in perovskite optoelectronic devices (Fig. 6).

\section{[H2] Exploiting disorder.}

The results discussed above raise the question, should one strive to entirely eliminate the heterogeneities? The point of view for conventional semiconductors is that uniform and invariant optoelectronic performance is essential for efficient operation, and this view has directed researchers to target monocrystalline materials ${ }^{117,183,184}$. Indeed, the negative impact that strain has on perovskite solar cell device performance and stability ${ }^{38,124,185,186}$ suggests that we should aim to eliminate these heterogeneous features. The elimination of these features by either reducing film stress and/or strain by precise thermal control during synthesis, or by appropriate substrate selection in order to match the thermal coefficients between the perovskite and substrate, has been shown to enhance perovskite device stability $^{124,185}$. Yet, carrier and phonon scattering mechanisms are still relatively unexplored, and how these processes are affected by local strain on the lattice remains an open question.

As strain is important for overall perovskite performance, lattice matching at the perovskite-transport layer heterojunctions ${ }^{185}$ must also be carefully considered. Owing to 
their 'soft' lattices and increased malleability, perovskites appear more tolerant of strain mismatching than most solar cell technologies ${ }^{15,66,187,188}$; however, the induced strain may have underlying consequences on device performance. For example, challenges arise in the vertical transport direction when the perovskite-transport layer connectivity is sub-optimal (Fig. 5a). To address this, passivation treatments are executed either pre- or post-deposition with varying degrees of effectiveness on the macroscopic device $\eta$; for example, incorporating larger molecules, such as phenethylammonium or guanidinium, into the perovskite structure at the interfaces with device contacts may relieve surface $\operatorname{strain}^{189,190}$. An exhaustive list of all the additives and passivation methods is beyond the scope of this Review; however, their influence on the performance and longevity of the optoelectronic properties of the perovskites is substantial ${ }^{8}$. Furthermore, many of the perovskite heterogeneities discussed thus far may also be mitigated, retarded or completely eliminated by rationally engineering additional chemical components ${ }^{22,77,125,130,191}$, layers or coatings ${ }^{192}$ to block and/or passivate problematic pathways ${ }^{193}$.

There are circumstances and applications, however, in which the presence of heterogeneity in halide perovskites may be advantageous. For example, strain fields ${ }^{194}$, which inhabit all length scales and directions (lateral and vertical), could be designed to funnel charges (or ions) to the regions where they are needed, enabling perovskites to be synthesized with specific strain pathways to control flow of charge carriers. In addition, the long-range supergrain features could be important for understanding why halide perovskites have excellent charge-carrier transport that extends well beyond the apparent grain size estimated by SEM. It may even be possible to efficiently transport charges over these super-grain entities, which opens up the possibility for efficient lateral or rear-contact solar cell devices ${ }^{195,196}$.

Apart from strain, heterogeneity-assisted performance has also been demonstrated by controlling charge-carrier flow within mixed-halide perovskites through self-organized 
bandgap gradients ${ }^{103}$. These gradients systematically promote charge-carrier accumulation in particular energy regions, leading to localized photodoping effects. Therefore, these areas with excess charge carriers may increase radiative recombination efficiencies and lead to radiative events outcompeting non-radiative pathways. Heterogeneity may also provide a mechanism to generate non-centrosymmetric (that is, piezoelectric) halide perovskite compositions, which could lead to ferroelectric properties and, in turn, to potential applications in optoelectronic devices that operate based on Rashba ${ }^{197,198}$ or flexophotovoltaic effects ${ }^{199}$. By exploiting such phenomena, researchers could engineer advantageous nanoscale heterogeneity and pave the way for the fabrication of perovskites with photovoltaic properties that approach or even surpass Shockley-Queisser limits. Additionally, the excellent charge-transport and sharp band-edge properties of halide perovskites despite notable disorder are reminiscent of liquid metals and appear to be unique for solid-state materials ${ }^{200-202}$.

Of course, such benefits of the heterogeneity need to be carefully considered on a circumstantial basis; there are clear situations when non-uniformities are undesirable (for example, pits in films, poor electrical contacts or the presence of other unwanted phases) and will undoubtedly be accompanied by a low $\eta$. Thus, we expect that heterogeneity will particularly benefit novel device designs. Nevertheless, even in present optoelectronic (for example, solar cell) architectures, there is only a minimal performance penalty for the presence of heterogeneity, and there may even be gains from local doping effects. Moreover, the increased processing costs associated with removing heterogeneity entirely may not be offset by apparent gains in performance or stability, suggesting there could be a compromise between the level of heterogeneity to balance performance, stability and facile processing.

Halide perovskites are an extraordinary material class that cannot be treated as a traditional semiconductor, because in doing so, we may ultimately limit its use rather than 
unlock its full potential. There is still much for us to discover about the fundamental nature of these intriguing semiconductors. As we develop a deeper understanding of their underlying material properties at multiple length scales through the advanced characterization methods that are now becoming available, we will fortify the next generation of perovskite optoelectronics.

\section{Acknowledgements}

E.M.T. acknowledges funding from the UK Engineering and Physics Sciences Research Council under grant reference EP/R023980/1. T.A.S.D. acknowledges support from a National University of Ireland Travelling Studentship. S.D.S. acknowledges the Royal Society and Tata Group (UF150033). The work has received funding from the European Research Council under the European Union's Horizon 2020 research and innovation programme (HYPERION, grant agreement No. 756962). The authors gratefully acknowledge helpful discussions with S. Macpherson, J. M. Howard, G. Hodes, D. Cahen and D. N. Johnstone. The authors also thank the Diamond Light Source for access and support in the use of the electron Physical Science Imaging Centre (instrument E02 and proposal numbers EM19793-1 and EM19793-2) that contributed to the data presented here.

\section{Author contributions}

All authors contributed equally to the preparation of this manuscript.

\section{Competing interests}

S.D.S. is a co-founder of Swift Solar, a company commercializing high-power, lightweight perovskite solar panels. E.M.T. and T.A.S.D. declare no competing interests. 


\section{Publisher's note}

Springer Nature remains neutral with regard to jurisdictional claims in published maps and institutional affiliations.

\section{References}

1 Yu, P. Y. \& Cardona, M. in Fundamentals of Semiconductors: Physics and Materials Properties Ch. 4, (Springer Berlin Heidelberg, 2010).

2 Pierret, R. F. in Advanced Semiconductor Fundamentals Vol. 6 Ch. 5, (Prentice Hall, 2003).

3 Stoneham, A. M. Non-radiative transitions in semiconductors. Reports on Progress in Physics 44, 1251 (1981).

4 Queisser, H. J. \& Haller, E. E. Defects in semiconductors: Some fatal, some vital. Science 281, 945-950 (1998).

5 Childress, L. \& Hanson, R. Diamond NV centers for quantum computing and quantum networks. MRS Bulletin 38, 134-138 (2013).

6 Monroe, C. Quantum information processing with atoms and photons. Nature 416, 238 (2002).

7 NREL Efficiency Chart. Best research-cell efficiency chart, https://www.nrel.gov/pv/assets/pdfs/best-research-cell-efficiencies-190416.pdf (2019).

8 Wang, F., Bai, S., Tress, W., Hagfeldt, A. \& Gao, F. Defects engineering for highperformance perovskite solar cells. npj Flexible Electronics 2, 22 (2018).

9 Leijtens, T. et al. Carrier trapping and recombination: the role of defect physics in enhancing the open circuit voltage of metal halide perovskite solar cells. Energy Environ. Sci. 9, 34723481 (2016).

10 Stranks, S. D. et al. Electron-hole diffusion lengths exceeding 1 micrometer in an organometal trihalide perovskite absorber. Science 342, 341-344 (2013).

11 Brandt, R. E. et al. Searching for "Defect-Tolerant" Photovoltaic Materials: Combined Theoretical and Experimental Screening. Chemistry of Materials 29, 4667-4674 (2017).

12 Agiorgousis, M. L., Sun, Y.-Y., Zeng, H. \& Zhang, S. Strong covalency-induced recombination centers in perovskite solar cell material $\mathrm{CH}_{3} \mathrm{NH}_{3} \mathrm{PbI}_{3}$. J. Am. Chem. Soc. 136, 14570-14575 (2014).

13 Yin, W.-J., Shi, T. \& Yan, Y. Unusual defect physics in $\mathrm{CH}_{3} \mathrm{NH}_{3} \mathrm{PbI}_{3}$ perovskite solar cell absorber. Appl. Phys. Lett. 104, 063903 (2014).

14 Kim, J., Lee, S.-H., Lee, J. H. \& Hong, K.-H. The role of intrinsic defects in methylammonium lead iodide perovskite. J. Phys. Chem. Lett. 5, 1312-1317 (2014).

15 Ball, J. M. \& Petrozza, A. Defects in perovskite-halides and their effects in solar cells. Nature Energy 1, 1-13 (2016).

16 Yin, W.-J., Shi, T. \& Yan, Y. Unique properties of halide perovskites as possible origins of the superior solar cell performance. Adv. Mater. 26, 4653-4658 (2014).

17 Zakutayev, A. et al. Defect tolerant semiconductors for solar energy conversion. J. Phys. Chem. Lett. 5, 1117-1125 (2014).

$18 \mathrm{Li}, \mathrm{W}$. et al. Chemically diverse and multifunctional hybrid organic-inorganic perovskites. Nat. Rev. Mater. 2, 16099 (2017).

19 Luo, Y. et al. The relationship between chemical flexibility and nanoscale charge collection in hybrid halide perovskites. Adv. Funct. Mater. 28, 1706995 (2018).

20 Poindexter, J. R. et al. High tolerance to iron contamination in lead halide perovskite solar cells. ACS Nano 11, 7101-7109 (2017).

21 Luo, Y. et al. Spatially heterogeneous chlorine incorporation in organic-inorganic perovskite solar cells. Chem. Mater. 28, 6536-6543 (2016). 
22 Abdi-Jalebi, M. et al. Maximizing and stabilizing luminescence from halide perovskites with potassium passivation. Nature 555, 497 (2018).

23 Liang, J. et al. Enhancing optical, electronic, crystalline, and morphological properties of cesium lead halide by Mn substitution for high-stability all-inorganic perovskite solar cells with carbon electrodes. Adv. Energy Mater. 8, 1800504 (2018).

24 Saidaminov, M. I. et al. Planar-integrated single-crystalline perovskite photodetectors. Nat. Commun. 6, 8724 (2015).

25 Ran, C., Xu, J., Gao, W., Huang, C. \& Dou, S. Defects in metal triiodide perovskite materials towards high-performance solar cells: origin, impact, characterization, and engineering. Chem. Soc. Rev. 47, 4581-4610 (2018).

26 Xing, G. et al. Low-temperature solution-processed wavelength-tunable perovskites for lasing. Nat. Mater. 13, 476 (2014).

27 Lee, J. W., Shichijo, H., Tsai, H. L. \& Matyi, R. J. Defect reduction by thermal annealing of GaAs layers grown by molecular beam epitaxy on Si substrates. Appl. Phys. Lett. 50, 31-33 (1987).

28 Stranks, S. D. Nonradiative losses in metal halide perovskites. ACS Energy Lett. 2, 1515-1525 (2017).

29 Kayes, B. M. et al. in 2011 37th IEEE Photovoltaic Specialists Conference. 000004-000008.

30 Jiang, Q. et al. Surface passivation of perovskite film for efficient solar cells. Nat. Photonics, (2019).

31 Yablonovitch, E., Miller, O. D. \& Kurtz, S. R. in 2012 38th IEEE Photovoltaic Specialists Conference. 001556-001559.

32 Shockley, W. \& Queisser, H. J. Detailed balance limit of efficiency of $\mathrm{p}-\mathrm{n}$ junction solar cells. Journal of Applied Physics 32, 510-519 (1961).

33 Tennyson, E. M. et al. Nanoimaging of open-circuit voltage in photovoltaic devices. Adv. Energy Mater. 5, 1501142 (2015).

34 Tennyson, E. M. et al. Caesium-incorporated triple cation perovskites deliver fully reversible and stable nanoscale voltage response. ACS Nano 13, 1538-1546 (2019).

35 Rothmann, M. U. et al. Direct observation of intrinsic twin domains in tetragonal $\mathrm{CH}_{3} \mathrm{NH}_{3} \mathrm{PbI}_{3}$. Nat. Commun. 8, 14547 (2017).

36 McKenna, K. P. Electronic properties of $\{111\}$ twin boundaries in a mixed-ion lead halide perovskite solar absorber. ACS Energy Lett. 3, 2663-2668 (2018).

37 Liu, Y. et al. Chemical nature of ferroelastic twin domains in $\mathrm{CH}_{3} \mathrm{NH}_{3} \mathrm{PbI}_{3}$ perovskite. Nat. Mater. 17, 1013-1019 (2018).

38 Jones, T. W. et al. Lattice strain causes non-radiative losses in halide perovskites. Energy Environ. Sci. 12, 596-606 (2019).

39 Phung, N. \& Abate, A. The impact of nano- and microstructure on the stability of perovskite solar cells. Small 14, 1802573 (2018).

40 Howard, J. M. et al. Humidity-induced photoluminescence hysteresis in variable $\mathrm{Cs} / \mathrm{Br}$ ratio hybrid perovskites. J. Phys. Chem. Lett. 9, 3463-3469 (2018).

41 Howard, J. M., Tennyson, E. M., Neves, B. R. \& Leite, M. S. Machine learning for perovskites' reap-rest-recovery cycle. Joule 3, 325-337 (2018).

42 Saliba, M., Correa-Baena, J. P., Gratzel, M., Hagfeldt, A. \& Abate, A. Perovskite solar cells: From the atomic level to film quality and device performance. Angewandte Chemie 57, 25542569 (2018).

43 Roose, B., Wang, Q. \& Abate, A. The role of charge selective contacts in perovskite solar cell stability. Adv. Energy Mater. 9, 1803140 (2018).

44 Bercegol, A. et al. Spatial inhomogeneity analysis of cesium-rich wrinkles in triple-cation perovskite. J. Phys. Chem. C 122, 23345-23351 (2018).

45 Bush, K. A. et al. Controlling thin-film stress and wrinkling during perovskite film formation. ACS Energy Lett. 3, 1225-1232 (2018).

46 Weber, S. A. L. et al. How the formation of interfacial charge causes hysteresis in perovskite solar cells. Energy Environ. Sci. 11, 2404-2413 (2018). 
47 Bowman, A. R. et al. Microsecond carrier lifetimes, controled p-doping and enhanced air stability in low-bandgap metal halide perovskites. In Review

$\mathrm{Bi}$, D. et al. Polymer-templated nucleation and crystal growth of perovskite films for solar cells with efficiency greater than 21\%. Nat. Energy 1, 16142 (2016).

Nayak, P. K. et al. Mechanism for rapid growth of organic-inorganic halide perovskite crystals. Nat. Commun. 7, 13303 (2016).

Tidhar, Y. et al. Crystallization of methyl ammonium lead halide perovskites: Implications for photovoltaic applications. J. Am. Chem. Soc. 136, 13249-13256 (2014).

Eperon, G. E. et al. The importance of moisture in hybrid lead halide perovskite thin film fabrication. ACS Nano 9, 9380-9393 (2015).

52 Gao, H. et al. Nucleation and crystal growth of organic-inorganic lead halide perovskites under different relative humidity. ACS Appl. Mater. Interfaces 7, $9110-9117$ (2015).

53 Rothmann, M. U. et al. Structural and chemical changes to $\mathrm{CH}_{3} \mathrm{NH}_{3} \mathrm{PbI}_{3}$ induced by electron and gallium ion beams. Adv. Mater. 30, 1800629 (2018).

54 Tian, W. et al. Limiting perovskite solar cell performance by heterogeneous carrier extraction. Angewandte Chemie 55, 13067-13071 (2016).

55 Zong, Y. et al. Continuous grain-boundary functionalization for high-efficiency perovskite solar cells with exceptional stability. Chem 4, 1404-1415 (2018).

56 Park, B.-w. et al. Understanding how excess lead iodide precursor improves halide perovskite solar cell performance. Nature Communications 9, 3301 (2018).

57 Kosasih, F. U. \& Ducati, C. Characterising degradation of perovskite solar cells through insitu and operando electron microscopy. Nano Energy 47, 243-256 (2018).

58 Tian, Y. et al. Enhanced organo-metal halide perovskite photoluminescence from nanosized defect-free crystallites and emitting sites. J. Phys. Chem. Lett. 6, 4171-4177 (2015).

59 Zhou, Y., Sternlicht, H. \& Padture, N. P. Transmission Electron Microscopy of Halide Perovskite Materials and Devices. Joule 3, 641-661 (2019).

60 Correa-Baena, J.-P. et al. Homogenized halides and alkali cation segregation in alloyed organic-inorganic perovskites. Science 363, 627-631 (2019).

61 Leblebici, S. Y. et al. Facet-dependent photovoltaic efficiency variations in single grains of hybrid halide perovskite. Nat. Energy 1, 16093 (2016).

62 Eperon, G. E., Moerman, D. \& Ginger, D. S. Anticorrelation between local photoluminescence and photocurrent suggests variability in contact to active layer in perovskite solar cells. ACS Nano 10, 10258-10266 (2016).

63 Parsons, S. Introduction to twinning. Acta Crystallogr. D 59, 1995-2003 (2003).

64 Strelcov, E. et al. $\mathrm{CH}_{3} \mathrm{NH}_{3} \mathrm{PbI}_{3}$ perovskites: Ferroelasticity revealed. Sci. Adv. 3, e1602165 (2017).

65 Rakita, Y. et al. Tetragonal $\mathrm{CH}_{3} \mathrm{NH}_{3} \mathrm{PbI}_{3}$ is ferroelectric. Proc. Natl. Acad. Sci. 114, E5504E5512 (2017).

66 Egger, D. A. et al. What remains unexplained about the properties of halide perovskites? Adv. Mater. 30, 1800691 (2018).

67 Zhang, D. et al. Atomic-resolution transmission electron microscopy of electron beamsensitive crystalline materials. Science 359, 675-679 (2018).

68 Chen, S. et al. Atomic scale insights into structure instability and decomposition pathway of methylammonium lead iodide perovskite. Nat. Commun. 9, 4807 (2018).

69 Egerton, R. F. Mechanisms of radiation damage in beam-sensitive specimens, for TEM accelerating voltages between 10 and 300 kV. Microscopy Research and Technique 75, 15501556 (2012).

70 Milosavljević, A. R., Huang, W., Sadhu, S. \& Ptasinska, S. Low-energy electron-induced transformations in organolead halide perovskite. Angewandte Chemie 128, 10237-10241 (2016).

71 Klein-Kedem, N., Cahen, D. \& Hodes, G. Effects of light and electron beam irradiation on halide perovskites and their solar cells. Acc. Chem. Res. 49, 347-354 (2016). 
72 Frost, J. M. et al. Atomistic origins of high-performance in hybrid halide perovskite solar cells. Nano Lett. 14, 2584-2590 (2014).

73 Frost, J. M., Butler, K. T. \& Walsh, A. Molecular ferroelectric contributions to anomalous hysteresis in hybrid perovskite solar cells. Appl. Phys. Lett. 2, 081506 (2014).

74 Wei, J. et al. Hysteresis analysis based on the ferroelectric effect in hybrid perovskite solar cells. J. Phys. Chem. Lett. 5, 3937-3945 (2014).

75 Garten, L. M. et al. The existence and impact of persistent ferroelectric domains in $\mathrm{MAPbI}_{3}$. Sci. Adv. 5, eaas9311 (2019).

76 Salje, E. K. J. A. R. o. M. R. Ferroelastic materials. Annual Review of Materials Research 42, 265-283 (2012).

77 deQuilettes, D. W. et al. Impact of microstructure on local carrier lifetime in perovskite solar cells. Science 348, 683-686 (2015).

78 deQuilettes, D. W. et al. Tracking Photoexcited Carriers in Hybrid Perovskite Semiconductors: Trap-Dominated Spatial Heterogeneity and Diffusion. ACS Nano 11, 1148811496 (2017).

79 Yang, M. et al. Do grain boundaries dominate non-radiative recombination in $\mathrm{CH}_{3} \mathrm{NH}_{3} \mathrm{PbI}_{3}$ perovskite thin films? Phys. Chem. Chem. Phys. 19, 5043-5050 (2017).

80 Zhou, Y., Game, O. S., Pang, S. \& Padture, N. P. Microstructures of organometal trihalide perovskites for solar cells: Their evolution from solutions and characterization. J. Phys. Chem. Lett. 6, 4827-4839 (2015).

81 Khassaf, H., Yadavalli, S. K., Zhou, Y., Padture, N. P. \& Kingon, A. I. Effect of Grain Boundaries on Charge Transport in Methylammonium Lead Iodide Perovskite Thin Films. $J$. Phys. Chem. C 123, 5321-5325 (2019).

82 Adhyaksa, G. W. P. et al. Understanding detrimental and beneficial grain boundary effects in halide perovskites. Adv. Mater. 30, 1804792 (2018).

83 Giesbrecht, N. et al. Synthesis of perfectly oriented and micrometer-sized $\mathrm{MAPbBr}_{3}$ perovskite crystals for thin-film photovoltaic applications. ACS Energy Lett. 1, 150-154 (2016).

84 Xing, J. et al. High-efficiency light-emitting diodes of organometal halide perovskite amorphous nanoparticles. ACS Nano 10, 6623-6630 (2016).

85 Wang, J. et al. Reducing surface recombination velocities at the electrical contacts will improve perovskite photovoltaics. ACS Energy Lett. 4, 222-227 (2019).

86 Reid, O. G., Yang, M., Kopidakis, N., Zhu, K. \& Rumbles, G. Grain-size-limited mobility in methylammonium lead iodide perovskite thin films. ACS Energy Lett. 1, 561-565 (2016).

87 Edri, E. et al. Elucidating the charge carrier separation and working mechanism of $\mathrm{CH}_{3} \mathrm{NH}_{3} \mathrm{PbI}_{3-\mathrm{x}} \mathrm{Cl}_{\mathrm{x}}$ perovskite solar cells. Nat. Commun. 5, 3461 (2014).

88 Barnard, E. S. et al. 3D lifetime tomography reveals how $\mathrm{CdCl}_{2}$ improves recombination throughout CdTe solar cells. Adv. Mater. 29, 1603801 (2016).

89 Ono, L. K. \& Qi, Y. Surface and Interface Aspects of Organometal Halide Perovskite Materials and Solar Cells. J. Phys. Chem. Lett. 7, 4764-4794 (2016).

90 Brenner, T. M., Egger, D. A., Kronik, L., Hodes, G. \& Cahen, D. Hybrid organic-inorganic perovskites: low-cost semiconductors with intriguing charge-transport properties. Nat. Rev. Mater. 1, 15007 (2016).

91 Walsh, A. \& Stranks, S. D. Taking control of ion transport in halide perovskite solar cells. ACS Energy Lett. 3, 1983-1990 (2018).

92 Stavrakas, C. et al. Probing buried recombination pathways in perovskite structures using 3D photoluminescence tomography. Energy Environ. Sci. 11, 2846-2852 (2018).

93 Ahn, N. et al. Trapped charge-driven degradation of perovskite solar cells. Nat. Commun. 7, 13422 (2016).

94 Yoon, S. J., Kuno, M. \& Kamat, P. V. Shift happens. How halide ion defects influence photoinduced segregation in mixed halide perovskites. ACS Energy Lett. 2, 1507-1514 (2017). 
95 Brennan, M. C., Draguta, S., Kamat, P. V. \& Kuno, M. Light-induced anion phase segregation in mixed halide perovskites. ACS Energy Lett. 3, 204-213 (2018).

Noh, J. H., Im, S. H., Heo, J. H., Mandal, T. N. \& Seok, S. I. Chemical management for colorful, efficient, and stable inorganic-organic hybrid nanostructured solar cells. Nano Lett. 13, 1764-1769 (2013).

97 Jesper Jacobsson, T. et al. Exploration of the compositional space for mixed lead halogen perovskites for high efficiency solar cells. Energy Environ. Sci. 9, 1706-1724 (2016).

98 Gratia, P. et al. Intrinsic halide segregation at nanometer scale determines the high efficiency of mixed cation/mixed halide perovskite solar cells. J. Am. Chem. Soc. 138, 15821-15824 (2016).

99 Song, T.-B., Sharp, I. D. \& Sutter-Fella, C. M. Understanding macroscale functionality of metal halide perovskites in terms of nanoscale heterogeneities. Journal of Physics: Energy 1, 011002 (2018).

100 Dou, L. et al. Spatially resolved multicolor $\mathrm{CsPbX}_{3}$ nanowire heterojunctions via anion exchange. Proc. Natl. Acad. Sci. 114, 7216-7221 (2017).

101 Dar, M. I. et al. Asymmetric cathodoluminescence emission in $\mathrm{CH}_{3} \mathrm{NH}_{3} \mathrm{PbI}_{3-\mathrm{x}} \mathrm{Br}_{\mathrm{x}}$ perovskite single crystals. ACS Photonics 3, 947-952 (2016).

102 Hentz, O., Zhao, Z. \& Gradečak, S. Impacts of ion segregation on local optical properties in mixed halide perovskite films. Nano Lett. 16, 1485-1490 (2016).

$103 \mathrm{Li}, \mathrm{W}$. et al. Phase segregation enhanced ion movement in efficient inorganic $\mathrm{CsPbIBr}_{2}$ solar cells. Adv. Energy Mater. 7, 1700946 (2017).

104 Kulbak, M. et al. Cesium enhances long-term stability of lead bromide perovskite-based solar cells. J. Phys. Chem. Lett. 7, 167-172 (2016).

105 Hoke, E. T. et al. Reversible photo-induced trap formation in mixed-halide hybrid perovskites for photovoltaics. Chem. Sci. 6, 613-617 (2015).

106 Edri, E. et al. Why lead methylammonium tri-Iodide perovskite-based solar cells require a mesoporous electron transporting scaffold (but not necessarily a hole conductor). Nano Lett. 14, 1000-1004 (2014).

107 Stranks, S. D. et al. Recombination kinetics in organic-inorganic perovskites: Excitons, free charge, and subgap states. Physical Review Applied 2, 034007 (2014).

108 Stranks, S. D., Hoye, R. L. Z., Di, D., Friend, R. H. \& Deschler, F. The physics of light emission in halide perovskite devices. Adv. Mater., 1803336 (2019).

109 Kovalenko, M. V., Protesescu, L. \& Bodnarchuk, M. I. Properties and potential optoelectronic applications of lead halide perovskite nanocrystals. Science 358, 745-750 (2017).

110 El-Hajje, G. et al. Quantification of spatial inhomogeneity in perovskite solar cells by hyperspectral luminescence imaging. Energy Environ. Sci. 9, 2286-2294 (2016).

111 Xiao, M. et al. A fast deposition-crystallization procedure for highly efficient lead iodide perovskite thin-film solar cells. Angewandte Chemie 126, 10056-10061 (2014).

112 Cho, N. et al. Pure crystal orientation and anisotropic charge transport in large-area hybrid perovskite films. Nat. Commun. 7, 13407 (2016).

113 Kim, D. H. et al. 300\% Enhancement of carrier mobility in uniaxial-oriented perovskite films formed by topotactic-oriented attachment. Adv. Mater. 29, 1606831 (2017).

114 Kim, M. K. et al. Effective control of crystal grain size in $\mathrm{CH}_{3} \mathrm{NH}_{3} \mathrm{PbI}_{3}$ perovskite solar cells with a pseudohalide $\mathrm{Pb}(\mathrm{SCN})_{2}$ additive. CrystEngComm 18, 6090-6095 (2016).

115 Kim, W. et al. Oriented grains with preferred low-angle grain boundaries in halide perovskite films by pressure-induced crystallization. Adv. Energy Mater. 8, 1702369 (2018).

116 Li, W., Fan, J., Mai, Y. \& Wang, L. Aquointermediate assisted highly orientated perovskite thin films toward thermally stable and efficient solar cells. Adv. Energy Mater. 7, 1601433 (2017).

117 Nie, W. et al. High-efficiency solution-processed perovskite solar cells with millimeter-scale grains. Science 347, 522-525 (2015). 
118 Sun, Y., Peng, J., Chen, Y., Yao, Y. \& Liang, Z. Triple-cation mixed-halide perovskites: towards efficient, annealing-free and air-stable solar cells enabled by $\mathrm{Pb}(\mathrm{SCN})_{2}$ additive. Sci. Rep. 7, 46193 (2017).

119 Yang, W. S. et al. High-performance photovoltaic perovskite layers fabricated through intramolecular exchange. Science 348, 1234-1237 (2015).

120 Tress, W. et al. Predicting the open-circuit voltage of $\mathrm{CH}_{3} \mathrm{NH}_{3} \mathrm{PbI}_{3}$ perovskite solar cells using electroluminescence and photovoltaic quantum efficiency spectra: The role of radiative and non-radiative recombination. Adv. Energy Mater. 5, 1400812 (2015).

121 Tvingstedt, K. et al. Radiative efficiency of lead iodide based perovskite solar cells. Sci. Rep. 4, 6071 (2014).

122 Würfel, P., Finkbeiner, S. \& Daub, E. J. A. P. A. Generalized Planck's radiation law for luminescence via indirect transitions. Applied Physics A 60, 67-70 (1995).

123 Stolterfoht, M. et al. Visualization and suppression of interfacial recombination for highefficiency large-area pin perovskite solar cells. Nature Energy, (2018).

124 Zhao, J. et al. Strained hybrid perovskite thin films and their impact on the intrinsic stability of perovskite solar cells. Sci. Adv. 3, (2017).

125 Wang, J. T.-W. et al. Efficient perovskite solar cells by metal ion doping. Energy Environ. Sci. 9, 2892-2901 (2016).

126 Lilliu, S. et al. Mapping morphological and structural properties of lead halide perovskites by scanning nanofocus XRD. Adv. Funct. Mater. 26, 8221-8230 (2016).

127 Saliba, M. et al. Cesium-containing triple cation perovskite solar cells: improved stability, reproducibility and high efficiency. Energy Environ. Sci. 9, 1989-1997 (2016).

128 Akkerman, Q. A. et al. Solution synthesis approach to colloidal cesium lead halide perovskite nanoplatelets with monolayer-level thickness control. J. Am. Chem. Soc. 138, 1010-1016 (2016).

129 Chu, Z. et al. Impact of grain boundaries on efficiency and stability of organic-inorganic trihalide perovskites. Nature Communications 8, 2230 (2017).

$130 \mathrm{Li}, \mathrm{T}$. et al. Additive engineering for highly efficient organic-inorganic halide perovskite solar cells: recent advances and perspectives. J Mater Chem A 5, 12602-12652 (2017).

131 Zhao, Y. et al. Perovskite seeding growth of formamidinium-lead-iodide-based perovskites for efficient and stable solar cells. Nature Communications 9, 1607 (2018).

132 Pathoor, N. et al. Fluorescence blinking beyond nanoconfinement: Spatially synchronous intermittency of entire perovskite microcrystals. Angewandte Chemie 130, 11777-11781 (2018).

133 Moerman, D., Eperon, G. E., Precht, J. T. \& Ginger, D. S. Correlating Photoluminescence Heterogeneity with Local Electronic Properties in Methylammonium Lead Tribromide Perovskite Thin Films. Chemistry of Materials 29, 5484-5492 (2017).

134 Tian, Y. et al. Giant photoluminescence blinking of perovskite nanocrystals reveals singletrap control of luminescence. Nano Lett. 15, 1603-1608 (2015).

135 Merdasa, A. et al. "Supertrap" at work: Extremely efficient nonradiative recombination channels in $\mathrm{MAPbI}_{3}$ perovskites revealed by luminescence super-resolution imaging and spectroscopy. ACS Nano 11, 5391-5404 (2017).

136 Gagliardi, A. \& Abate, A. Mesoporous electron-selective contacts enhance the tolerance to interfacial ion accumulation in perovskite solar cells. ACS Energy Lett. 3, 163-169 (2018).

137 Ravishankar, S. et al. Influence of charge transport layers on open-circuit voltage and hysteresis in perovskite solar cells. Joule 2, 788-798 (2018).

138 Simpson, M. J., Doughty, B., Yang, B., Xiao, K. \& Ma, Y.-Z. Spatial localization of excitons and charge carriers in hybrid perovskite thin films. J. Phys. Chem. Lett. 6, 3041-3047 (2015).

139 Pazos-Outón, L. M. et al. Photon recycling in lead iodide perovskite solar cells. Science 351, 1430-1433 (2016).

140 Dursun, I. et al. Efficient photon recycling and radiation trapping in cesium lead halide perovskite waveguides. ACS Energy Lett. 3, 1492-1498 (2018). 
141 Shimamura, K., Yuan, Z., Shimojo, F. \& Nakano, A. Effects of twins on the electronic properties of GaAs. Appl. Phys. Lett. 103, 022105 (2013).

142 Garrett, J. L. et al. Real-time nanoscale open-circuit voltage dynamics of perovskite solar cells. Nano Lett. 17, 2554-2560 (2017).

143 Jariwala, S. et al. Imaging grain structure in halide perovskites: local crystal misorientation influences non-radiative recombination. ArXiv, (2019).

144 Calais, E. et al. Tectonic strain in plate interiors? Nature 438, E9-E10 (2005).

145 Tsai, H. et al. Light-induced lattice expansion leads to high-efficiency perovskite solar cells. Science 360, 67-70 (2018).

146 Vrućinić, M. et al. Local Versus Long-Range Diffusion Effects of Photoexcited States on Radiative Recombination in Organic-Inorganic Lead Halide Perovskites. Adv. Sci. 2, (2015).

147 Kutes, Y. et al. Mapping the photoresponse of $\mathrm{CH}_{3} \mathrm{NH}_{3} \mathrm{PbI}_{3}$ hybrid perovskite thin films at the nanoscale. Nano Lett. 16, 3434-3441 (2016).

148 Bergmann, V. W. et al. Real-space observation of unbalanced charge distribution inside a perovskite-sensitized solar cell. Nat Commun 5, 5001 (2014).

149 deQuilettes, D. W. et al. Photo-induced halide redistribution in organic-inorganic perovskite films. Nat. Commun. 7, 11683 (2016).

150 Draguta, S. et al. Rationalizing the light-induced phase separation of mixed halide organicinorganic perovskites. Nat. Commun. 8, 200 (2017).

151 Nie, W. et al. Light-activated photocurrent degradation and self-healing in perovskite solar cells. Nat. Commun. 7, 11574 (2016).

152 Gottesman, R. et al. Extremely slow photoconductivity response of $\mathrm{CH}_{3} \mathrm{NH}_{3} \mathrm{PbI}_{3}$ perovskites suggesting structural changes under working conditions. J. Phys. Chem. Lett. 5, 2662-2669 (2014).

153 Barker, A. J. et al. Defect-assisted photoinduced halide segregation in mixed-halide perovskite thin films. ACS Energy Lett. 2, 1416-1424 (2017).

154 Nickel, N. H., Lang, F., Brus, V. V., Shargaieva, O. \& Rappich, J. Unraveling the LightInduced Degradation Mechanisms of $\mathrm{CH}_{3} \mathrm{NH}_{3} \mathrm{PbI}_{3}$ Perovskite Films. Adv. Electron. Mater. 3, 1700158 (2017).

155 Bischak, C. G. et al. Origin of reversible photoinduced phase separation in hybrid perovskites. Nano Lett. 17, 1028-1033 (2017).

$156 \mathrm{Xu}$, R.-P. et al. In Situ Observation of Light Illumination-Induced Degradation in Organometal Mixed-Halide Perovskite Films. ACS Appl. Mater. Interfaces 10, 6737-6746 (2018).

157 Liu, Z. et al. Open-circuit voltages exceeding $1.26 \mathrm{~V}$ in planar methylammonium lead iodide perovskite solar cells. ACS Energy Lett., 110-117 (2018).

158 Chen, S. et al. Light illumination induced photoluminescence enhancement and quenching in lead halide perovskite. Solar RRL 1, 1600001 (2017).

159 Brenes, R., Eames, C., Bulović, V., Islam, M. S. \& Stranks, S. D. The impact of atmosphere on the local luminescence properties of metal halide perovskite grains. Adv. Mater. 30, 1706208 (2018).

160 Tennyson, E. M., Howard, J. M. \& Leite, M. S. Mesoscale functional imaging of materials for photovoltaics. ACS Energy Lett. 2, 1825-1834 (2017).

$161 \mathrm{Li}, \mathrm{X}$. et al. Improved performance and stability of perovskite solar cells by crystal crosslinking with alkylphosphonic acid $\omega$-ammonium chlorides. Nat. Chem. 7, 703 (2015).

162 Bischak, C. G., Sanehira, E. M., Precht, J. T., Luther, J. M. \& Ginsberg, N. S. Heterogeneous charge carrier dynamics in organic-inorganic hybrid materials: Nanoscale lateral and depthdependent variation of recombination rates in methylammonium lead halide perovskite thin films. Nano Lett. 15, 4799-4807 (2015).

163 Osherov, A. et al. The Impact of Phase Retention on the Structural and Optoelectronic Properties of Metal Halide Perovskites. Adv. Mater. 28, 10757-10763 (2016). 
164 Galkowski, K. et al. Spatially resolved studies of the phases and morphology of methylammonium and formamidinium lead tri-halide perovskites. Nanoscale 9, 3222-3230 (2017).

165 Mahboubi Soufiani, A. et al. Impact of microstructure on the electron-hole interaction in lead halide perovskites. Energy Environ. Sci. 10, 1358-1366 (2017).

166 Divitini, G. et al. In situ observation of heat-induced degradation of perovskite solar cells. Nat. Energy 1, 15012 (2016).

167 Collins, L. et al. Breaking the time barrier in Kelvin probe force microscopy: Fast free force reconstruction using the g-mode platform. ACS Nano 11, 8717-8729 (2017).

168 Kutes, Y. et al. Direct Observation of Ferroelectric Domains in Solution-Processed CH3NH3PbI3 Perovskite Thin Films. The Journal of Physical Chemistry Letters 5, 33353339 (2014).

169 Chen, B. et al. Imaging Spatial Variations of Optical Bandgaps in Perovskite Solar Cells. Adv. Energy Mater. 0, 1802790 (2018).

170 Song, Z. et al. Perovskite solar cell stability in humid air: Partially reversible phase transitions in the $\mathrm{PbI}_{2}-\mathrm{CH}_{3} \mathrm{NH}_{3} \mathrm{I}-\mathrm{H}_{2} \mathrm{O}$ system. Adv. Energy Mater. 6, 1600846 (2016).

171 Harvey, S. P. et al. Probing perovskite inhomogeneity beyond the surface: TOF-SIMS analysis of halide perovskite photovoltaic devices. ACS Appl. Mater. Interfaces 10, 2854128552 (2018).

172 Quintero-Bermudez, R. et al. Compositional and orientational control in metal halide perovskites of reduced dimensionality. Nature Materials 17, 900-907 (2018).

173 Brenes, R. et al. Metal halide perovskite polycrystalline films exhibiting properties of single crystals. Joule 1, 155-167 (2017).

174 Anaya, M., Galisteo-López, J. F., Calvo, M. E., Espinós, J. P. \& Míguez, H. Origin of lightinduced photophysical effects in organic metal halide perovskites in the presence of oxygen. J. Phys. Chem. Lett. 9, 3891-3896 (2018).

175 Tian, Y. et al. Mechanistic insights into perovskite photoluminescence enhancement: light curing with oxygen can boost yield thousandfold. Phys. Chem. Chem. Phys. 17, 24978-24987 (2015).

176 Midgley, P. \& Johnstone, D. Scanning Electron Diffraction - Crystal Mapping at the Nanoscale. Microscopy and Microanalysis 24, 182-183 (2018).

177 de la Pena, F. et al. Electron microscopy (big and small) data analysis with the open source software package HyperSpy. Microscopy and Microanalysis 23, 214-215 (2017).

178 Johnstone, D. N. et al. (2019, April 24) pyxem/pysem: pyXem 0.7.1 (Version v0.7.1)Zenodo. http://doi.org/10.5281/zenodo.2650296

179 Butler, K. T., Davies, D. W., Cartwright, H., Isayev, O. \& Walsh, A. Machine learning for molecular and materials science. Nature 559, 547-555 (2018).

180 Borodinov, N. et al. Deep neural networks for understanding noisy data applied to physical property extraction in scanning probe microscopy. npj Computational Materials 5, 25 (2019).

181 Cacovich, S. et al. Unveiling the chemical composition of halide perovskite films using multivariate statistical analyses. ACS Appl. Energy Mater. 1, 7174-7181 (2018).

182 Martineau, B. H., Johnstone, D. N., van Helvoort, A. T. J., Midgley, P. A. \& Eggeman, A. S. Unsupervised machine learning applied to scanning precession electron diffraction data. Advanced Structural and Chemical Imaging 5, 3 (2019).

183 Grancini, G. et al. $\mathrm{CH}_{3} \mathrm{NH}_{3} \mathrm{PbI}_{3}$ perovskite single crystals: surface photophysics and their interaction with the environment. Chem. Sci. 6, 7305-7310 (2015).

184 Huang, J., Shao, Y. \& Dong, Q. Organometal trihalide perovskite single crystals: A next wave of materials for $25 \%$ efficiency photovoltaics and applications beyond? J. Phys. Chem. Lett. 6, 3218-3227 (2015).

185 Rolston, N. et al. Engineering stress in perovskite solar cells to improve stability. Adv. Energy Mater. 8, 1802139 (2018). 
186 Li, X., Luo, Y., Holt, M. V., Cai, Z. \& Fenning, D. P. Residual Nanoscale Strain in Cesium Lead Bromide Perovskite Reduces Stability and Shifts Local Luminescence. Chem. Mater. 31, 2778-2785 (2019).

187 Shi, H. \& Du, M.-H. Shallow halogen vacancies in halide optoelectronic materials. Phys. Rev. $B$ 90, 174103 (2014).

188 Lai, M. et al. Intrinsic anion diffusivity in lead halide perovskites is facilitated by a soft lattice. Proc. Natl. Acad. Sci. 115, 11929-11934 (2018).

189 Rajagopal, A., Stoddard, R. J., Jo, S. B., Hillhouse, H. W. \& Jen, A. K. Y. Overcoming the photovoltage plateau in large bandgap perovskite photovoltaics. Nano Lett. 18, 3985-3993 (2018).

190 Stoddard, R. J. et al. Enhancing defect tolerance and phase stability of high-bandgap perovskites via guanidinium alloying. ACS Energy Lett. 3, 1261-1268 (2018).

191 Yang, W. S. et al. Iodide management in formamidinium-lead-halide-based perovskite layers for efficient solar cells. Science 356, 1376-1379 (2017).

$192 \mathrm{Li}, \mathrm{B}$. et al. Surface passivation engineering strategy to fully-inorganic cubic $\mathrm{CsPbI} 3$ perovskites for high-performance solar cells. Nature Communications 9, 1076 (2018).

193 Zhang, W. et al. Enhanced optoelectronic quality of perovskite thin films with hypophosphorous acid for planar heterojunction solar cells. Nat. Commun. 6, 10030 (2015).

194 Zhu, C. et al. Strain engineering in perovskite solar cells and its impacts on carrier dynamics. Nat. Commun. 10, 815 (2019).

195 Hou, Q. et al. Back-contact perovskite solar cells with honeycomb-like charge collecting electrodes. Nano Energy 50, 710-716 (2018).

196 Lin, X. et al. Effect of grain cluster size on back-contact perovskite solar cells. Adv. Funct. Mater. 28, 1805098 (2018).

197 Stranks, S. D. \& Plochocka, P. The influence of the Rashba effect. Nat. Mater. 17, 381-382 (2018).

198 Kim, M., Im, J., Freeman, A. J., Ihm, J. \& Jin, H. Switchable S=1/2 and J=1/2 Rashba bands in ferroelectric halide perovskites. Proc. Natl. Acad. Sci. 111, 6900-6904 (2014).

199 Yang, M.-M., Kim, D. J. \& Alexe, M. Flexo-photovoltaic effect. Science 360, 904-907 (2018).

200 Faber, T. E. \& Ziman, J. M. A theory of the electrical properties of liquid metals. The Philosophical Magazine: A Journal of Theoretical Experimental and Applied Physics 11, 153-173 (1965).

201 Guo, Y. et al. Liquid-like free carrier solvation and band edge luminescence in lead-halide perovskites. ArXiv, arXiv:1804.01509 (2018).

202 Zhu, H. et al. Screening in crystalline liquids protects energetic carriers in hybrid perovskites.

Science 353, 1409-1413 (2016).

Fig. 1 Comparison of the photovoltaic properties of GaAs and perovskite devices. a $\mid$ Current densityvoltage curves show that both GaAs $\left(\right.$ ref. $\left.{ }^{29}\right)$ and halide perovskite $\left(\left(\mathrm{FA}_{0.85} \mathrm{MA}_{0.15}\right) \mathrm{PbI}_{3} ;\right.$ ref. $\left.^{30}\right)$ solar cells exhibit large macroscopic open-circuit voltages $\left(V_{\text {oc }}\right)$. The inset shows the unit cells of GaAs and a perovskite. Perovskites adopt an $\mathrm{ABX}_{3}$ structure, where $\mathrm{A}^{+}$is a cation (such as methylammonium, formamidinium or $\mathrm{Cs}^{+}$), $\mathrm{B}^{2+}$ is a metal (such as $\mathrm{Pb}^{2+}$ and/or $\left.\mathrm{Sn}^{2+}\right)$ and $\mathrm{X}^{-}$is a halide $\left(\mathrm{I}^{-}, \mathrm{Br}^{-}\right.$and/or $\mathrm{Cl}^{-}$). b,c $\mid$Despite GaAs and perovskites having similar $V_{\mathrm{oc}}$ values, the local distribution in $V_{\mathrm{oc}}\left(\Delta V_{\mathrm{oc}}\right)$ at the nanoscale are remarkably different. The $\Delta V_{\text {oc }}$ map of a typical GaAs sample (panel b), acquired using Kelvin probe force microscopy ${ }^{33}$, reveals that GaAs has a uniform voltage response. By contrast, perovskites display large voltage variations (panel c) despite their high performance. The perovskite $\Delta V_{\text {oc }}$ map was obtained in-house on a $\left(\mathrm{Cs}_{0.05} \mathrm{FA}_{0.8} \mathrm{MA}_{0.15}\right) \mathrm{Pb}\left(\mathrm{I}_{0.83} \mathrm{Br}_{0.17}\right)_{3}$ perovskite with comparable device performance to that in panel a. Panel $\mathbf{a}$ is adapted with permission from ref. ${ }^{29}$, IEEE, and ref. ${ }^{30}$, Springer Nature Limited. Panel $\mathbf{b}$ is adapted with permission from ref. ${ }^{33}$, Wiley-VCH.

Fig. 2 The hierarchy of heterogeneity in halide perovskites. a-c | The electron microscopy images exemplify the sub-grain (panel a), grain-to-grain (panel b) and long-range (panel c) disorder within halide 
perovskites. d | A cross-sectional scanning electron microscopy image of a complete high-performance perovskite solar cell. Vertical heterogeneity in the perovskite grain morphology is evident despite the device having an excellent power conversion efficiency of $23.32 \%$. $\mathrm{FA}^{+}$, formamidinium; ITO, indium-doped tin oxide; $\mathrm{MA}^{+}$, methylammonium; spiro-OMeTAD, 2,2',7,7'-tetrakis( $N, N$-di- $p$-methoxyphenylamine)-9, $9^{\prime}-$ spirobifluorene. Panel $\mathbf{c}$ is adapted with permission from ref. ${ }^{44}$, ACS. Panel $\mathbf{d}$ is adapted from ref. ${ }^{30}$, Springer Nature Limited.

Fig. 3 | Nanoscale sub-grain heterogeneity. a | Open-circuit voltage $\left(V_{\text {oc }}\right)$ map of a $\mathrm{MAPbI}_{3-x} \mathrm{Cl}_{x}$ (where $\mathrm{MA}^{+}$ is methylammonium) film showing facet-dependent heterogeneity in the electronic properties of the halide perovskite on the sub-grain scale. $\mathbf{b} \mid$ The top part illustrates the segregation of $\mathrm{I}^{-}$and $\mathrm{Cs}^{+}$to a $\{111\}$ twin boundary (TB, dashed line) in $\mathrm{FA}_{0.875} \mathrm{Cs}_{0.125} \mathrm{~Pb}\left(\mathrm{I}_{0.666} \mathrm{Br}_{0.333}\right)_{3}$ (where $\mathrm{FA}^{+}$is formamidinium). The bottom part shows the predicted density of states for the bulk and twin boundary regions of the perovskite; the purple shaded areas in the top part indicate the regions used in the projected density-of-state calculations. c | The top part shows a high-resolution transmission electron microscopy image of a $\mathrm{MAPbI}_{3}$ single crystal; the squares indicate two ordered domains with off-centred $\mathrm{MA}^{+}$cations in two different orientations. The bottom part shows the structural models and the simulated projected potential maps of $\mathrm{MAPbI}_{3}$ with $\mathrm{MA}^{+}$adopting parallel (left) and normal (right) orientations with respect to the projection direction. $\mathbf{d} \mid$ The band excitation piezoresponse force microscopy (BE-PFM) frequency image (top) reveals twin domain regions with varying elasticity in a $\mathrm{MAPbI}_{3}$ sample. The corresponding helium ion microscopy coupled with secondary ion mass spectroscopy chemical map (bottom) shows the spatial distribution of $\mathrm{MA}^{+}$in the $\mathrm{MAPbI}_{3}$ sample; the black arrows indicate chemically segregated twin domains. $E$, energy; $E_{\mathrm{F}}$, Fermi level. Panel a is adapted from ref. ${ }^{61}$, Springer Nature Limited. Panel $\mathbf{b}$ is adapted with permission from ref. ${ }^{36}$, ACS. Panel $\mathbf{c}$ is adapted with permission from ref. ${ }^{67}$, AAAS. Panel $\mathbf{d}$ is adapted from ref. ${ }^{37}$, Springer Nature Limited.

Fig. 4 | Grain-to-grain heterogeneity. a | Photoluminescence (PL) maps of $\mathrm{MAPbI}_{3}$ (where $\mathrm{MA}^{+}$is methylammonium) films at different photon fluxes. The incident excitation intensity influences the transport mechanisms of the charge carriers: at low intensity (left), the charge carriers are trap limited, whereas at higher intensities (right), the charge carriers are diffusion limited. b | Scanning electron microscopy (SEM) images (top) and electron back-scattering diffraction (EBSD) plots (bottom) of $\mathrm{MAPbBr}_{3}$ perovskite thin-film samples with different grain sizes (stated above the corresponding SEM image). The heterogeneity in the crystallographic orientation increases with grain size, and amorphous grain boundaries (white regions) are present in the sample with $32-\mu \mathrm{m}$-sized grains. Note that the colours in the EBSD plots do not represent a specific crystallographic orientation. $\mathbf{c}$ The plot of yield-mobility product as a function of grain size in a $\mathrm{MAPbI}_{3}$ perovskite shows that the mobility decreases as the average grain size decreases. The symbols represent the experimental data (with error bars indicating the standard deviation in both the grain size and yield-mobility product). The dashed line is the best fit of the Kubo relationship for the first two data points, whereas the solid line is the best fit for all the data. d | Single-photon (left) and two-photon (right) PL maps that probe the surface and bulk radiative recombination emission, respectively, in a $\mathrm{MAPbI}_{3}$ thin film. e $\mid$ Spectrally integrated cathodoluminescence (CL) maps of a $\mathrm{CsPbIBr}_{2}$ film acquired in an electron microscope. The top part shows the high-energy spectrum, which is probably from a $\mathrm{Br}^{-}$-rich phase, whereas the middle part shows lower-energy emission and is likely from an $\mathrm{I}^{-}$-rich phase. The bottom map is a superposition of the two CL images (green indicates high-energy emission, and red indicates lower-energy emission) and shows that the $\mathrm{I}^{-}$-rich regions are pushed toward the grain boundaries. f | A PL intensity map (left) of a $\mathrm{MAPbI}_{3}$ thin film overlaid with a fluorescence-lifetime imaging microscopy map. The brightness indicates the PL emission intensity, whereas the colour indicates the lifetime. Three grains are emphasized with respect to their neighbours by line profiles L1, L2 and L3; each of these three grains has a low PL intensity compared with its neighbouring grains (top right part). However, as indicated by the line traces on the bottom right, the central grains in L1, L2 and L3 exhibit sustained, longer or shorter lifetimes, respectively, than the neighbouring grains. Panel a is adapted with permission from ref. ${ }^{78}$, ACS. Panel $\mathbf{b}$ is adapted with permission from ref. ${ }^{82}$, Wiley-VCH. Panel $\mathbf{c}$ is adapted with permission from ref. ${ }^{86}$, ACS. Panel $\mathbf{d}$ is adapted with permission from ref. ${ }^{92}$, RSC. Panel $\mathbf{e}$ is adapted with permission from ref. ${ }^{103}$, Wiley-VCH. Panel $\mathbf{f}$ is adapted with permission from ref. ${ }^{79}$, RSC.

Fig. 5 | Long-range heterogeneity in halide perovskites. a | Map of the quasi-Fermi level splitting $(\Delta \mu)$ for a $\mathrm{MAPbI}_{3}$ (where $\mathrm{MA}^{+}$is methylammonium) perovskite device, quantified by using the generalized Planck's law. The large regions of lower $\Delta \mu$ are probably due to poor electrical contact between the electron-transport and perovskite layers. $\mathbf{b} \mid$ The left part shows the time-resolved photoluminescence (PL) decay for two regions of a $\mathrm{MAPbI}_{3}$ film. The bright and dark regions are highlighted by the blue and red circles, respectively, in the micro $\mathrm{X}$-ray diffraction map shown on the right. Regions with less strain have a longer PL lifetime. $\mathbf{c} \mid$ In situ PL maps 
showing the growth of a $\mathrm{Cs}_{0.10} \mathrm{FA}_{0.78} \mathrm{MA}_{0.12} \mathrm{PbI}_{2.55} \mathrm{Br}_{0.45}$ (where $\mathrm{FA}^{+}$is formamidinium) film at different time points. The PL map of the seed- $-\mathrm{PbI}_{2}$ film is shown on the left. Deposition of organic ammonium salts onto the seed- $-\mathrm{PbI}_{2}$ film initiates crystallization of the perovskite film (middle and right maps). The halide perovskite sample demonstrates better material quality (that is, higher PL emission) in regions where a nucleation seed is embedded (indicated by white circles in the middle map). The red circle in the middle map indicates a region of random nucleation without a seed. d | The top part shows a PL map of fused $\mathrm{MAPbBr}_{3}$ grains (I-IV) and an isolated $\mathrm{MAPbBr}_{3}$ grain (V). The bottom part shows the associated PL time traces for the fused grains and the isolated grain. The traces reveal that there is communication and synchronous PL blinking in the conjoined grains. Panel $\mathbf{a}$ is adapted with permission from ref. ${ }^{110}$, RSC. Panel $\mathbf{b}$ is adapted with permission from ref. ${ }^{38}$, RSC. Panel $\mathbf{c}$ is adapted from ref. ${ }^{131}$, Springer Nature Limited. Panel $\mathbf{d}$ is adapted with permission from ref. ${ }^{132}$, Wiley-VCH.

Fig. 6 Roadmap for implementing correlative microscopy for halide perovskites. Despite the numerous challenges associated with multimodal approaches, there are ways to overcome them, yielding valuable discoveries. For example, such measurements will reveal the complicated relationships between structural, chemical and electrical properties at the nanoscale, as well as quantify the tolerable dose limits for perovskites to external stimuli (such as bias, photons, electrons and X-rays) before generation of additional defects and hence non-radiative loss pathways. We anticipate that multimodal imaging will unveil new avenues to exploit halide perovskite non-uniformities. ETM, electron-transport material; HTM, hole-transport material. 